\title{
ELECTRON HEAT FLUX IN THE SOLAR WIND: ARE WE OBSERVING THE COLLISIONAL LIMIT IN THE 1 AU DATA?
}

\author{
S. LAndi ${ }^{1}$, L. Matteini ${ }^{2}$, And F. PANTEllini ${ }^{3}$ \\ ${ }^{1}$ Dipartimento di Fisica e Astronomia Università degli Studi di Firenze Largo E. Fermi 2, I-50125 Firenze, Italy \\ ${ }^{2}$ The Blackett Laboratory, Imperial College London Prince Consort Road, London SW7 2AZ, UK \\ ${ }^{3}$ LESIA, Observatoire de Paris, CNRS, UPMC, Université Paris-Diderot 5, place J. Janssen, F-92195 Meudon Cedex, France \\ Received 2014 March 27; accepted 2014 June 26; published 2014 July 9
}

\begin{abstract}
Using statistically significant data at $1 \mathrm{AU}$, it has recently been shown (Bale et al.) that in the solar wind, when the Knudsen number $K_{\mathrm{T}}$ (the ratio between the electron mean free path and the electron temperature scale height) drops below about 0.3 , the electron heat flux $q$ intensity rapidly approaches the classical collisional Spitzer-Härm limit. Using a fully kinetic model including the effect of Coulomb collisions and the expansion of the solar wind with heliocentric distance, we observe that the heat flux strength does indeed approach the collisional value for Knudsen numbers smaller than about 0.3 in very good agreement with the observations. However, closer inspection of the heat flux properties, such as its variation with the heliocentric distance and its dependence on the plasma parameters, shows that for Knudsen numbers between 0.02 and 0.3 the heat flux is not conveniently described by the Spitzer-Härm formula. We conclude that even though observations at $1 \mathrm{AU}$ seem to indicate that the electron heat flux intensity approaches the collisional limit when the Knudsen drops below $\sim 0.3$, the collisional limit is not a generally valid closure for a Knudsen larger than 0.01 . Moreover, the good agreement between the heat flux from our model and the heat flux from solar wind measurements in the high-Knudsen number regime seems to indicate that the heat flux at $1 \mathrm{AU}$ is not constrained by electromagnetic instabilities as both wave-particle and wave-wave interactions are neglected in our calculations.
\end{abstract}

Key words: methods: numerical - plasmas - solar wind

Online-only material: color figures

\section{INTRODUCTION}

Electron heat conduction plays an important role in determining the electron energy budget in the expanding solar wind, and hence how much heating by external sources (e.g., wave turbulence) is needed to account for the properties of the electrons in the solar wind (see, e.g., Cranmer et al. 2009). Despite the Knudsen number $K_{\mathrm{T}}$ (the ratio between the mean-free path and the gradient scale of the macroscopic quantities) being of the order of unity in the solar wind, theoretical studies (see Landi et al. 2012, and references therein) and in situ observations (e.g., Salem et al. 2003; Štverák et al. 2008) have shown that collisions play an important role in shaping the electron velocity distribution function (eVDF), and hence determining the characteristics and evolution of macroscopic quantities such as the temperature and heat flux.

Several studies have shown that the classical valid formula for a collisional plasma (Spitzer \& Härm 1953) is not adequate for the electron heat flux in the weakly collisional, high-Knudsen solar wind. In the framework of laser heated plasma, Gray \& Kilkenny (1980) showed that the Spitzer-Härm's treatment of thermal conductivity breaks for Knudsen numbers of the order of 0.01. Bell et al. (1981) and Shvarts et al. (1981) showed that deviations from Spitzer-Härm occur for Knudsen numbers as small as $\sim 10^{-3}$. In a remarkable work on the solar transition region, Shoub (1983) argued that the validity of the collisional description is limited to the even more restrictive range of $K_{\mathrm{T}} \lesssim 10^{-3}$. Kinetic numerical simulations of the acceleration region of the wind (Landi \& Pantellini 2001) have confirmed that the heat flux is not adequately described by the collisional formula for $K_{\mathrm{T}} \simeq 10^{-2}$, a limit recently reaffirmed by a detailed theoretical analysis of the effect of the collisions on the different parts of the eVDF (Scudder \& Karimabadi 2013).

On the other hand, using a statistically significant database from the WIND spacecraft, Bale et al. (2013, hereafter B13) have shown that when the Knudsen number at $1 \mathrm{AU}$ drops below $\sim 0.3$, the heat flux makes a rather sudden transition from the collisionless to the collisional regime. The conclusion of Bale et al. (2013) is that in the solar wind, electron heat conduction is conveniently described by the collisional formula up to $\left(K_{\mathrm{T}} \approx 0.3\right)$, thus contradicting many of the above cited works.

In a recent work (Landi et al. 2012, hereafter L12), using a model that takes into account the effect of wind expansion and Coulomb binary collisions between particles, we were able to reproduce remarkably well several features of the solar wind electrons including their temperature profiles, temperature anisotropy, core-halo relative drift velocities and densities, as well as the global structure of the electron velocity distribution function. As only one of the simulations in L12 appeared to potentially be in the collisional limit, we decided to extend the number of simulations toward smaller Knudsen numbers in order to verify if a transition at $K_{\mathrm{T}}=0.3$ does exist in the simulations. We will see that such a transition does effectively appear in our simulations. However, the transition is not a sharp one. Thus, even though in the range $K_{\mathrm{T}} \simeq[0.02,0.3]$ the electron heat flux appears to rather closely follow the collisional prediction, it effectively preserves the typical properties of a non-collisional heat flux (i.e., its dependence on the electron density). Only for very low values of $K_{\mathrm{T}}$ (rather unlikely in the real solar wind at $1 \mathrm{AU}$ ) is a convergence toward the collisional limit effectively observed. 

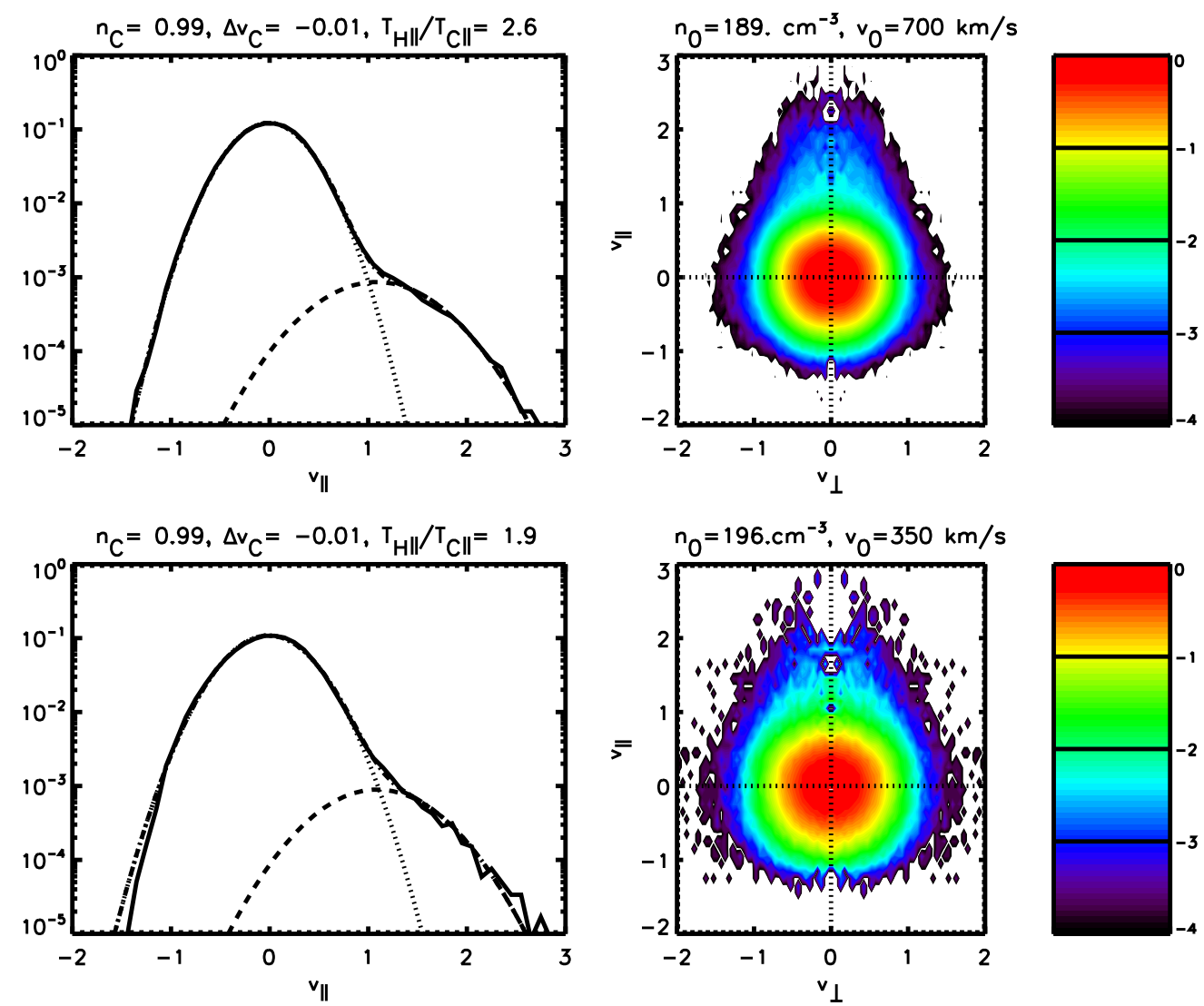

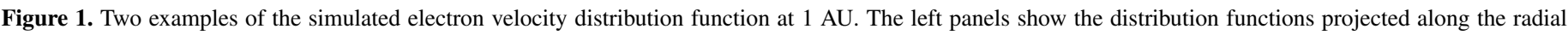

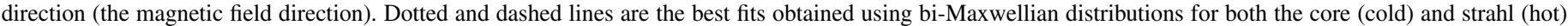

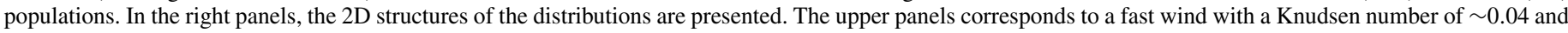
the bottom panels to a slow wind with a Knudsen number of $\sim 0.05$.

(A color version of this figure is available in the online journal.)

\section{SIMULATIONS}

For this study, we use a one-dimensional fully kinetic code including (1) binary Coulomb collisions between particles (electrons and ions), (2) supersonic radial expansion, and (3) a self-consistent interplanetary electric field ensuring quasineutrality and zero current. Details of the code can be found in previous works (Pantellini 2000; Landi \& Pantellini 2001, 2003).

For the set of simulations presented here, we use the same initial and boundary conditions adopted in L12. The inner boundary is located near $0.3 \mathrm{AU}$. The magnetic field is radial. The injected eVDF is a drifting isotropic Maxwellian with a temperature of $T_{\mathrm{e}}=1.5 \times 10^{5} \mathrm{~K}$ (Schwenn 1990). For protons, we choose a drifting bi-Maxwellian with a mean temperature of $T_{\mathrm{p}}=3 \times 10^{5} \mathrm{~K}$ and temperature anisotropy (with respect to the magnetic field) of $A_{\mathrm{p}}=T_{\mathrm{p} \perp} / T_{\mathrm{p} \|}=2$ (Matteini et al. 2007). As in L12, we choose a fast wind with a typical velocity of $700 \mathrm{~km} \mathrm{~s}^{-1}$ and a slow wind $\left(350 \mathrm{~km} \mathrm{~s}^{-1}\right)$. The simulation domain covers the radial interval $[0.3,3] \mathrm{AU}$. In L12, the range of the density $n_{0}$ at the base of the domain was restricted to the range 3 to $50 \mathrm{~cm}^{-3}$. Here, we present a set of 20 simulations where $n_{0}$ spans from about 2 to about $450 \mathrm{~cm}^{-3}$, in order to cover a larger interval of collision frequencies (and hence Knudsen numbers). The values used here go beyond the realistic values observed in the solar wind but allow us to cover a larger interval in parameter space (for example, we simulate exceptionally high density fast winds). For each simulation, collisions, radial expansion, and the self-consistent electric field shape the electron velocity distribution function throughout the simulation domain. The radial dependence of density, velocity, pressures, and heat flux is obtained by computing the moments of the velocity distribution function at various spatial locations.

Typical electron velocity distribution functions obtained in the simulations are shown in Figure 1. As already shown in L12, their shape and structure are similar to their counterparts in the solar wind. They exhibit a cold and almost isotropic core (containing most of the electrons) and a hot population drifting with respect to the core strongly beamed along the (radial) magnetic field, whose properties are very similar to those of the so-called "strahl" often observed in the solar wind and which carries most of the heat flux.

In the left panel of Figure 2, we show the ratio of the measured heat flux to the saturation heat flux as a function of the temperature-based Knudsen number for all simulations at three different heliocentric distances: $1,1.5$, and $2 \mathrm{AU}$. The saturation heat flux $q_{0}$ is defined as

$$
q_{0}=\frac{3}{2} n_{\mathrm{e}} k_{\mathrm{B}} T_{\mathrm{e}} v_{\mathrm{e}}
$$

where $n_{\mathrm{e}}$ is the total electron number density, $v_{\mathrm{e}}=\sqrt{2 k_{\mathrm{B}} T_{\mathrm{e}} / m_{\mathrm{e}}}$ is the electron thermal velocity, and $k_{\mathrm{B}}$ is the Boltzmann constant. The Knudsen number is defined as the ratio between the electron mean-free path $\lambda_{\mathrm{e}}$ and the electron temperature 

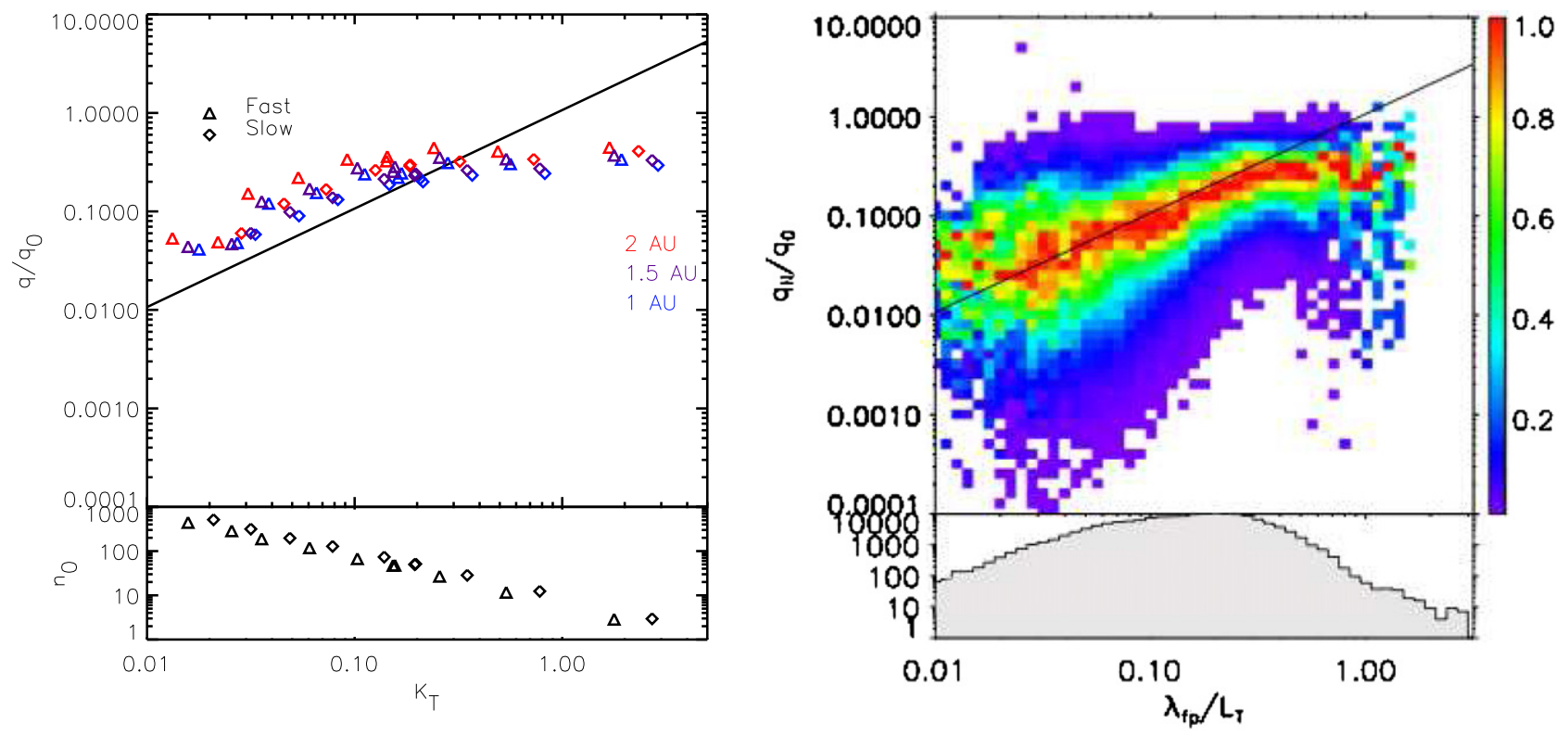

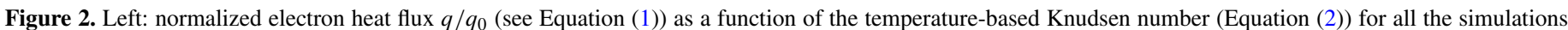

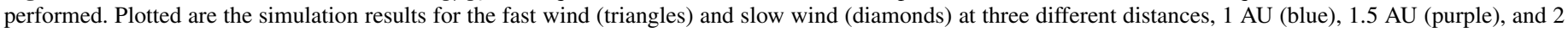

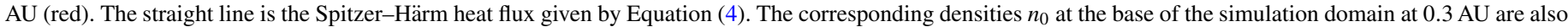
reported in the bottom panel. Right: WIND spacecraft data. (Figure 1 from Bale et al. 2013). The data histogram is also shown at the bottom.

(A color version of this figure is available in the online journal.)

gradient, namely,

$$
K_{\mathrm{T}}=\lambda_{\mathrm{e}} \frac{\left|\nabla T_{\mathrm{e}}\right|}{T_{\mathrm{e}}} .
$$

In the right panel of the figure, we report results from the WIND spacecraft (Figure 1 of B13). Simulation results and spacecraft measurements are remarkably similar. For both cases, we observe that in the domain $K_{\mathrm{T}} \gtrsim 0.1-0.2$, the normalized heat flux is roughly constant and independent on the Knudsen number. As already discussed (L12), the saturation level appears to increase slightly with distance while keeping roughly one order of magnitude below the saturation flux $q_{0}$. A similar transition near $K_{\mathrm{T}} \approx 0.1$ has been observed in the FokkerPlanck calculations by (Bell et al. 1981). We note that since our model does not include wave-particle interactions, the observed saturation cannot be ascribed to the effect of electromagnetic waves generated by microinstabilities. For $K_{\mathrm{T}} \lesssim 0.3$, the normalized heat flux becomes correlated with the Knudsen number keeping only slightly above the Spitzer-Härm flux (Braginskii 1965):

$$
q_{\mathrm{SH}}=-3.2 \frac{n_{\mathrm{e}} T_{\mathrm{e}} k_{\mathrm{B}}^{2}}{m_{\mathrm{e}} v_{\mathrm{ep}}} \nabla T,
$$

where $m_{\mathrm{e}}$ is the electron mass and $v_{\mathrm{ep}}$ is the electron collision frequency. Using Equations (1) and (2), and recalling that $v_{\mathrm{ep}}=v_{\mathrm{e}} / \lambda_{\mathrm{e}}$, we have

$$
\frac{\left|q_{\mathrm{SH}}\right|}{q_{0}}=1.07 K_{\mathrm{T}} .
$$

Both slow and fast streams are used to explore a Knudsen number domain large enough to cover the interval from $10^{-2}$ to $\sim 3$. However, Figure 2 shows that even though an increase of the wind velocity from 350 to $700 \mathrm{~km} \mathrm{~s}^{-1}$ induces a slight displacement toward smaller Knudsen numbers of the normalized heat flux versus $K_{\mathrm{T}}$ profile, the overall profile of the latter is not significantly altered by the change of velocity. Thus, while admittedly not universal, we propose that the heat flux versus $K_{\mathrm{T}}$ dependence revealed in Figure 2 is sufficiently generic to apply to most of the possible solar wind configurations.

The view presented in Figure 2 seems to confirm the conclusion that near $K_{\mathrm{T}} \approx 0.1$ there is an abrupt transition from a collisionless to a collisionally dominated heat flux (B13). In order to disprove this hypothesis, we draw the radial profiles of the heat flux for a set of simulations. In Figure 3, the solid lines represent the simulated radial heat flux profiles with different colors referring to different solar wind densities $n_{0}$. As a reference, for each simulation, the dashed profiles represent the corresponding collisional heat flux given by Equation (3). First, we note that the measured heat flux intensity varies with density as for a collisionless flux (e.g., Hollweg 1974; Landi \& Pantellini 2001), whereas, as expected, the Spitzer-Härm computed heat flux is rather insensitive to density. Second, since in all simulations the radial electron temperature gradients are similar, the corresponding collisional heat flux does not vary significantly from one simulation to the other in clear disagreement with the measured heat flux profiles in the simulations, even for the densest cases corresponding to Knudsen numbers $K_{\mathrm{T}}=0.02-0.03$ (red lines of Figure 3). We note, however, a progressive steepening of the observed heat flux profiles as the density increases, illustrating the gradual convergence toward the collision dominated profile.

Another way to appreciate the transition from collisionless to collisional is to compare the heat flux measured in the simulations with Hollweg's collisionless model for the heat flux in the solar wind (Hollweg 1974):

$$
q_{\mathrm{h}}=\alpha_{\mathrm{h}} \frac{3}{2} n_{\mathrm{e}} k_{\mathrm{B}} T_{\mathrm{e}} w_{0},
$$

where $w_{0}$ is the wind velocity and $\alpha_{\mathrm{h}}$ is a dimensionless coefficient of the order of unity. In L12, we have shown that Hollweg's expression applies relatively well to winds with 


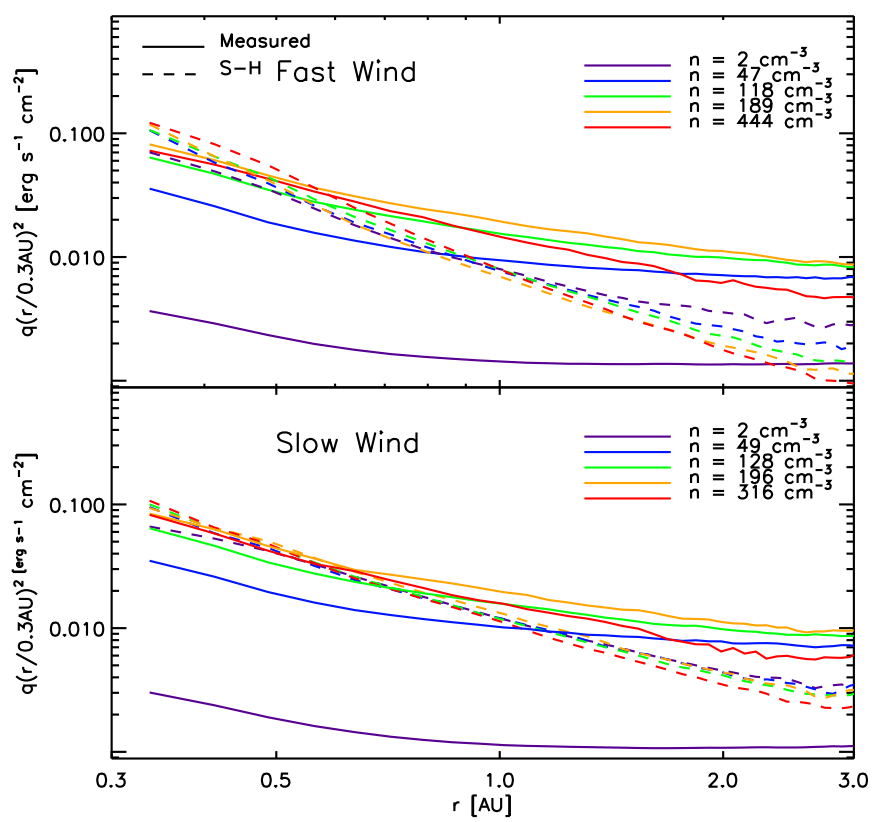

Figure 3. Simulated heat flux profiles compensated by $r^{2}$ as a function of heliocentric distance (solid lines). Colors denote various densities $n_{0}$ at the lower simulation boundary at 0.3 AU ranging from $2 \mathrm{~cm}^{-3}$ to about $450 \mathrm{~cm}^{-3}$. For each simulation, the dashed lines represent the heat flux computed from the collisional formula Equation (3). Top and bottom panels refer to simulations with fast and slow wind, respectively.

(A color version of this figure is available in the online journal.)

Knudsen numbers larger than $\sim 0.2$. In Figure 4, we report the radial variation of $\alpha_{\mathrm{h}}$ for a set of fast and slow wind simulations. The plots confirm that $\alpha_{\mathrm{h}}$ is not that different for the three most tenuous winds in either the fast or slow regime. Given that the density difference between the three most tenuous winds is $\sim 24$, the near constancy of $\alpha_{\mathrm{h}}$ indicates that in this regime the heat flux is essentially a Hollweg type, i.e., proportional to density. On the other hand, as the base density $n_{0}$ grows beyond $\sim 100 \mathrm{~cm}^{-3}$, $\alpha_{\mathrm{h}}$ starts to become density dependent indicating that Hollweg's collisionless model no longer provides an adequate description of the heat flux, once more suggesting that a transition toward a collisional regime is taking place. We finally note that a spatially constant value of $\alpha_{\mathrm{h}}$ in Figure 4 is not proof of the collisionless nature of the heat flux, but merely that $q \propto T / r^{2}$, which is also compatible with a Spitzer-Härm flux in a $T(r) \propto r^{-2 / 5}$ temperature profile.

\section{CONCLUSION}

Using a fully kinetic solar wind model which takes into account the effect of Coulomb collisions, we have studied the radial evolution of the electron heat flux for different wind speeds and densities. The simulations cover a sufficiently extended range of Knudsen numbers to include, at least in part, the transition from collisional to collisionless heat flux regime. The obtained results are in excellent agreement with solar wind measurements at $1 \mathrm{AU}$ (Bale et al. 2013).

For sufficiently large Knudsen numbers (larger than 0.1-0.2), the saturation flux normalized heat flux becomes Knudsen independent. In this regime, the heat flux peaks at a significant fraction (typically 0.2 to 0.3 ) of the saturation heat flux. Since in our model we do not take into account wave-particle and wave-wave interactions, we argue that the saturation level of the heat flux observed in the solar wind is not regulated by

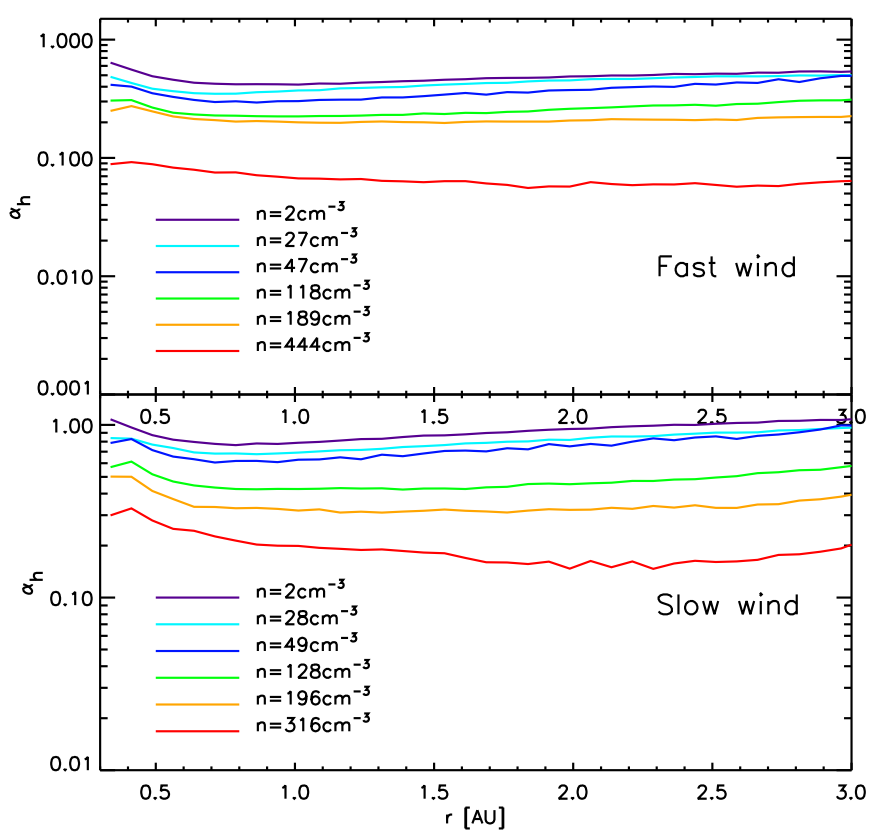

Figure 4. Heat flux normalized to $(3 / 2) n_{\mathrm{e}} k_{\mathrm{B}} T_{\mathrm{e}} w_{0}$ for a set of fast wind simulations $\left(w_{0}=750 \mathrm{~km} \mathrm{~s}^{-1}\right.$, upper panel) and slow wind simulations $\left(w_{0}=350 \mathrm{~km} \mathrm{~s}^{-1}\right.$, bottom panel).

(A color version of this figure is available in the online journal.)

electromagnetic instabilities. For decreasing Knudsen numbers, from 0.2 down to the smallest simulated case 0.02 , the normalized heat flux decreases following a path close to (but above) the path corresponding to the collisional flux Spitzer \& Härm (1953). These results may, at first sight, suggest that the heat flux is collisional in winds with $K_{\mathrm{T}} \lesssim 0.2$. Closer inspection of the heat flux radial variation and wind density dependence in our simulations shows that the heat flux is still not collision dominated for $K_{\mathrm{T}} \approx 0.02$. The transition in the normalized heat flux near $K_{\mathrm{T}}=0.2$ in Figure 2 merely indicates the beginning of the transition toward the collision-dominated regime, not the beginning of the collision-dominated regime. These findings agree with theoretical predictions (e.g., Shoub 1983; Scudder \& Karimabadi 2013) where the transition toward the collisional regime is expected for Knudsen numbers not exceeding 0.01. For completeness, we point out that as thoroughly discussed by Scudder \& Karimabadi (2013), the relevant Knudsen number defining the transition from the collisional to collisionless regime in the solar wind should be based on the electron pressure gradient rather than the thermal gradient as it is the former that provides the dominant contribution to the electric field in the solar wind. This is unlike the case in the transition region (discussed in Shoub 1983) where the electric field is dominated by the thermoelectric effect. The pressure scale length $\left|\left(\partial_{r} \ln p_{\mathrm{e}}\right)^{-1}\right|$ in the solar wind being $(2+\beta) / \beta \approx 3.5$ times shorter than the thermal scale length $\left|\left(\partial_{r} \ln T_{\mathrm{e}}\right)^{-1}\right|=\beta_{\mathrm{e}} / r$ for a typical solar wind value of $\beta_{\mathrm{e}}=0.8$, using the pressure Knudsen $K_{\mathrm{e}}$ in place of $K_{\mathrm{T}}$ in Figure 2 would merely shift all of the points and curves horizontally by a factor of 3.5 , leaving the overall structure unchanged. If the pressure gradient, = rather than the thermal gradient had to be adopted as the canonical reference for describing the transition, then it may well be that the measured points in Figure 2 are somewhat smeared out along $K_{\mathrm{T}}$ due to $\beta_{\mathrm{e}}$ slightly changing from one simulation to the other. Our choice to plot the normalized heat flux against the thermal 
Knudsen (rather than the pressure Knudsen) has the enormous advantage of allowing for a direct comparison of the measured flux with respect to the strongly collisional limit and also to facilitate the comparison with previous experimental works on the subject.

Since it was possible to recover the observed properties of the electron heat flux, using simulations where only Coulomb collisions and geometrical effects are taken into account, these results also enforce our conclusions from a previous work (Landi et al. 2012) where we pointed out that currently available measurements in the solar wind are insufficient to disentangle the effects of direct heating of the plasma from external sources (waves, turbulence, magnetic reconnection) and the shaping of the electron distribution functions due to radial expansion and Coulomb collisions. New and high-resolution measurements from the upcoming heliospheric missions Solar Orbiter and Solar Probe Plus will be helpful in understanding and constraining the physics of solar wind electrons.

The research leading to these results received funding from the European Commission's Seventh Framework Programme (FP7/2007-2013) under grant agreement SHOCK (project No. 284515). It was also supported by the UK Science and Technology Facilities Council grant ST/K001051/1. The authors thank the participants to the SOWHAT workshop held in Prague, 2013 July 19-21, for useful discussions.

\section{REFERENCES}

Bale, S. D., Pulupa, M., Salem, C., Chen, C. H. K., \& Quataert, E. 2013, ApJL, 769, L22

Bell, A. R., Evans, R. G., \& Nicholas, D. J. 1981, PhRvL, 46, 243

Braginskii, S. I. 1965, in Reviews of Plasma Physics, Vol. 1, ed. M. A. Leontovich (New York: Consultants Bureau), 205

Cranmer, S. R., Matthaeus, W. H., Breech, B. A., \& Kasper, J. C. 2009, ApJ, 702,1604

Gray, D. R., \& Kilkenny, J. D. 1980, PIPh, 22, 81

Hollweg, J. V. 1974, JGR, 79, 3845

Landi, S., Matteini, L., \& Pantellini, F. 2012, ApJ, 760, 143

Landi, S., \& Pantellini, F. 2003, A\&A, 400, 769

Landi, S., \& Pantellini, F. G. E. 2001, A\&A, 372, 686

Matteini, L., Landi, S., Hellinger, P., et al. 2007, GeoRL, 34, 20105

Pantellini, F. G. E. 2000, AmJPh, 68, 61

Salem, C., Hubert, D., Lacombe, C., et al. 2003, ApJ, 585, 1147

Schwenn, R. 1990, Large-Scale Structure of the Interplanetary Medium, Vol. 1 (1st ed.; Berlin: Springer), 99

Scudder, J. D., \& Karimabadi, H. 2013, ApJ, 770, 26

Shoub, E. C. 1983, ApJ, 266, 339

Shvarts, D., Delettrez, J., McCrory, R. L., \& Verdon, C. P. 1981, PhRvL, 47,247

Spitzer, L., \& Härm, R. 1953, PhRv, 89, 977

Stverák, Š., Trávníček, P., Maksimovic, M., et al. 2008, JGRA, 113, 3103 